\title{
Synthesis of imidazo[1,2-a] pyridines by rearrangement of 2-pyridyl-3-arylaminoisoxazol-5-(2H)-ones
}

\author{
Jabbar Khalafy ${ }^{*}$, Ali Reza Molla Ebrahimlo, Ronak Eisavi, and Karim Akbari Dilmaghani \\ Department of Chemistry, Urmia University, Urmia 57159, Iran \\ E-mail:jKhalafi@yahoo.com; J.Khalafi@mail.urmia.ac.ir
}

\begin{abstract}
2-Pyridyl-3-arylaminoisoxazol-5(2H)-ones, substituted on $\mathrm{N}$ with a nitropyridine group 12a-12e reacted with triethylamine in ethanol under reflux to give imidazo[1,2-a]pyridines 13a-13e and carbon dioxide. An analogous synthesis failed to yield ethyl 3-(2-ethoxycarbonylphenyl) amino5-oxo-2,5-dihydroisoxazole-4-carboxylate; the reaction of diethyl (2ethoxycarbonylphenyl)thiocarbamoylmalonate with hydroxylamine gave the novel isoxazolo[3,2-b]quinazoline $\mathbf{1 4}$ by intramolecular acylation of the isoxazolone intermediate 14a.
\end{abstract}

Keywords: Isoxazolones, 2-chloro-5-nitropyridine, rearrangements, triethylamine, imidazopyridines, isoxazoloquinazoline

\section{Introduction}

The reaction of 3-substituted isoxazolones with bases is not well known, and the only examples appear to be those reported by Doleschall ${ }^{1}$, who alkylated the anion of ethyl 2,3-dimethyl-2,5dihydro-5-oxo-isoxazole-4-carboxylate 1 in order to obtain $\gamma$-alkylated acetoacetates.

We have recently reported ${ }^{2}$ that the reaction of 2-aryl-3-arylaminoisoxazolones 2 with triethylamine leads to the formation of indoles 3 and carbon dioxide, an outcome that is formally the same as that achieved by photolysis. ${ }^{3}$ The evidence for the indole structure, rather than that of an isomer, rested on the number of aryl proton signals visible in the ${ }^{1} \mathrm{H}-\mathrm{NMR}$ spectrum.

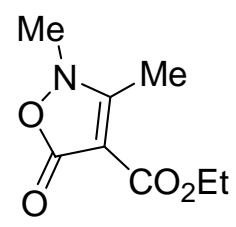

1<smiles>CCOC(=O)c1c(Nc2ccccc2)n([Al])oc1=O</smiles>

2<smiles>CCNc1[nH]c2ccccc2c1C(=O)OCC</smiles>

3 
We have recently reported ${ }^{2}$ that the reaction of 2-aryl-3-phenylaminoisoxazolones 2 substituted on nitrogen with an isoquinoline or quinazoline group, react with triethylamine to give imidazo annelated compounds 4 and 5, respectively. When the N-substituent is a nitropyridine the 2-aminoindole structure $\mathbf{6}$ was assigned to the product.<smiles>CCOC(=O)c1c(Nc2ccccc2)nc2c3ccccc3ccn12</smiles>

4<smiles>CCOC(=O)c1c(Nc2ccccc2)nc2c3ccccc3nc(-c3ccccc3)n12</smiles>

5<smiles>CCOC(=O)c1c(Nc2ccc([N+](=O)[O-])cn2)[nH]c2ccccc12</smiles>

6

We have also reported ${ }^{4}$ that arylaminoisoxazol-5(2H)-one 7 , substituted on nitrogen with a benzothiazole group 8 , reacts with triethylamine in ethanol under reflux conditions to provide a convenient synthesis of ethyl 2-arylaminoimidazo[2,1-b]benzothiazole-3-carboxylates 9 .<smiles>[R]OCc1c(Nc2cccc([R])c2)[nH]oc1=O</smiles>

7<smiles>[R]OCc1c(Nc2cccc([R])c2)n(-c2nc3ccccc3s2)oc1=O</smiles>

8<smiles>[R]OC(=O)c1c(Nc2cccc([R])c2)nc2sc3ccccc3n12</smiles>

9

$\mathrm{R}=\mathrm{H}, m-\mathrm{Me}, m-\mathrm{Br}$

Prager and co-workers have reported ${ }^{5}$ that 2-aryl-3-aminoisoxazol-5(2H)-ones undergo solvolysis to form 1,3-dipoles that undergo intermolecular cyclisation to form either imidazopyridines or indoles in the presence of potassium carbonate. The mode of cyclisation is controlled by the electronegativity of the aryl substituent.

Here we report the synthesis of 2-pyridyl-3-arylaminoisoxazol-5(2H)-ones 12a-12e, where the $\mathrm{N}$-substituent is a nitropyridine, and their reactions with triethylamine to form imidazopyridines 13a-13e, respectively. We also report the formation of isoxazolo[3,2b]quinazoline 14 by intramolecular acylation of the isoxazolone intermediate $14 a$. 


\section{Results and Discussion}

The required isoxazolones 12a-12e were synthesised by $\mathrm{N}$-arylation of the $2 \mathrm{H}$-isoxazolones 11a11e which in turn were made by a modification of the procedure of Worrall. ${ }^{6,7}$ Thus, the reaction of the sodium salt of diethyl malonate in ethanol with aryl isothiocyanates gave the thiocarbamates 10a-10e in good yield, and these were converted to the corresponding isoxazolones 11a-11e by reaction with hydroxylamine (Scheme 1).

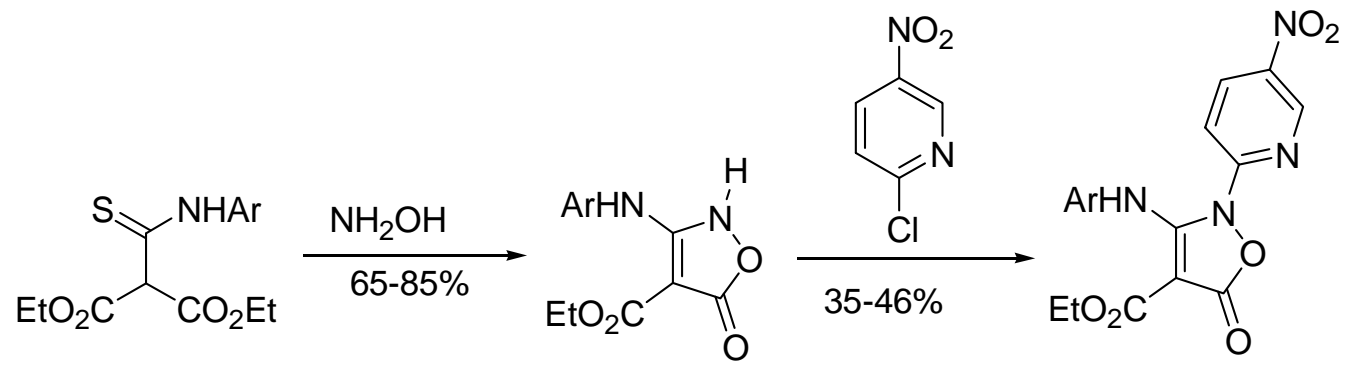

10

11

12

(a) $\mathrm{Ar}=3-\mathrm{BrC}_{6} \mathrm{H}_{4}$, (b) $\mathrm{Ar}=3-\mathrm{MeC}_{6} \mathrm{H}_{4}$, (c) $\mathrm{Ar}=4-\mathrm{NO}_{2} \mathrm{C}_{6} \mathrm{H}_{4}$, (d), $\mathrm{Ar}=3-\left(\mathrm{CO}_{2} \mathrm{Et}\right) \mathrm{C}_{6} \mathrm{H}_{4}$,

(e) $\mathrm{Ar}=4-\left(\mathrm{CO}_{2} \mathrm{Et}\right) \mathrm{C}_{6} \mathrm{H}_{4}$

\section{Scheme 1}

$\mathrm{N}$-Arylation of 11a-11e with 2-chloro-5-nitropyridine then gave the desired starting materials 12a-12e (Scheme I). While the formations of 12a-12e appear trivial, the reaction generally proceeded best in the absence of solvent, by heating the required reagent under nitrogen at $130^{\circ} \mathrm{C}$ for an hour.

The rearrangement of 2-pyridyl-5-isoxazolones 12a-12e proceeded in refluxing ethanol for 3$24 \mathrm{~h}$ in the presence of triethylamine (Scheme 2).

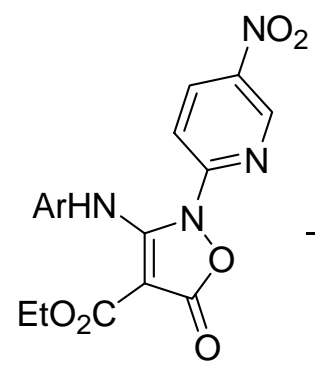

12

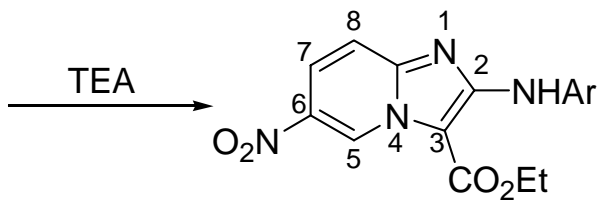

13

(a) $\mathrm{Ar}=3-\mathrm{BrC}_{6} \mathrm{H}_{4}$, (b) $\mathrm{Ar}=3-\mathrm{MeC}_{6} \mathrm{H}_{4}$, (c) $\mathrm{Ar}=4-\mathrm{NO}_{2} \mathrm{C}_{6} \mathrm{H}_{4}$, (d) $\mathrm{Ar}=3-\left(\mathrm{CO}_{2} \mathrm{Et}\right) \mathrm{C}_{6} \mathrm{H}_{4}$

(e) $\mathrm{Ar}=4-\left(\mathrm{CO}_{2} \mathrm{Et}\right) \mathrm{C}_{6} \mathrm{H}_{4}$

\section{Scheme 2}


Compound 12c reacted more slowly, but gave the corresponding imidazopyridine in $72 \%$ yield. It has been reported ${ }^{5}$ that the rearrangement of $12 \mathrm{c}$ in the presence of potassium carbonate in ethanol, returned mainly starting materials.

With a number of imidazopyridine structures in hand, the structures of all imidazopyridines were confirmed by ${ }^{1} \mathrm{HNMR},{ }^{13} \mathrm{CNMR}$, FT-IR, MASS spectra and microanalyses.

All compounds 13a-13e showed $\mathrm{H}-7$ to have meta coupling with $\mathrm{H}-5$, but the resonance for H-5 could not be clearly observed in compounds 13a-13e. The reason for the extreme broadening of this peak is unknown, though quadrupole coupling with N-4 is implicated. It has been reported previously ${ }^{8}$ that the X-ray structure of $(\mathbf{1 3}, \mathrm{Ar}=\mathrm{Ph})$ did not show any unusual interactions. The reaction pathway resulting in the imidazopyridines is consistent with our earlier suggestion (Scheme 3). ${ }^{2}$

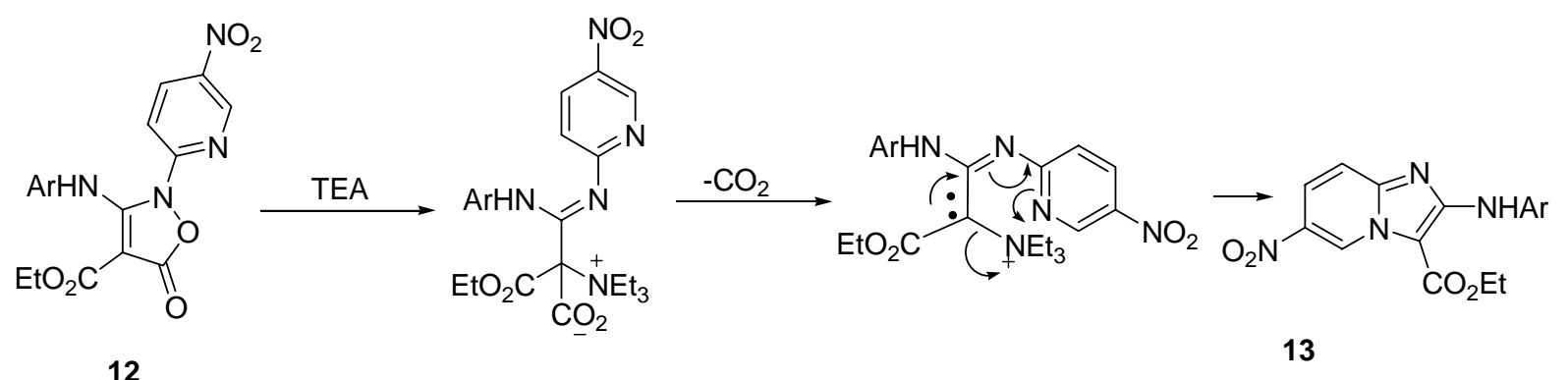

(13a) $\mathrm{Ar}=3-\mathrm{BrC}_{6} \mathrm{H}_{4}$, (13b) $\mathrm{Ar}=3-\mathrm{MeC}_{6} \mathrm{H}_{4}$, (13c) $\mathrm{Ar}=4-\mathrm{NO}_{2} \mathrm{C}_{6} \mathrm{H}_{4},\left(\right.$ 13d) $\mathrm{Ar}=3-\left(\mathrm{CO}_{2} \mathrm{Et}\right) \mathrm{C}_{6} \mathrm{H}_{4}$ (13e) $\mathrm{Ar}=4-\left(\mathrm{CO}_{2} \mathrm{Et}\right) \mathrm{C}_{6} \mathrm{H}_{4}$

\section{Scheme 3}

Therefore, these base induced rearrangements appear to be generally applicable to the synthesis of imidazopyridines, which are suitable synthetic intermediates for a series of polycyclic heterocycles.

It is interesting that the reaction of $\mathbf{1 0 f}$ with hydroxylamine gave ethyl 2,9-dioxo-4,9dihydro-2H-isoxazolo[3,2-b]quinazoline 14 as white needles $(81 \%)$, m.p. 266-268 ${ }^{\circ}$, by intramolecular acylation of the isoxazolone intermediate 14a (Scheme 4).

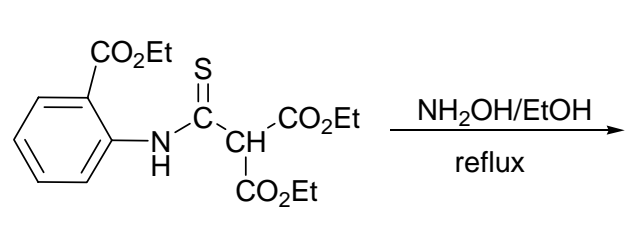

$10 f$

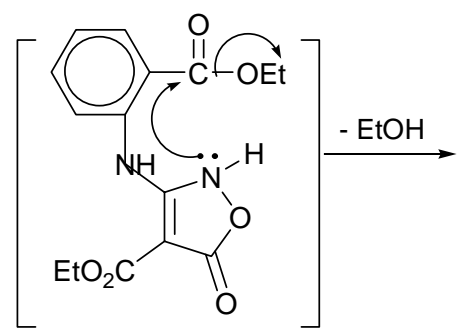

$14 a$

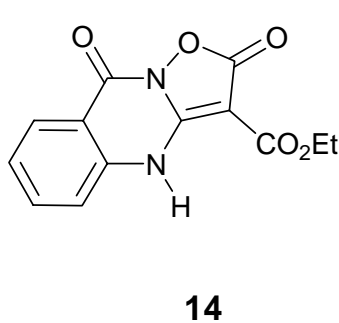

14

\section{Scheme 4}

The structure of 14 was confirmed by ${ }^{1} \mathrm{HNMR},{ }^{13} \mathrm{CNMR}$, FT-IR spectra and microanalysis. 


\section{Conclusions}

The rearrangement of the 2-pyridyl-3-arylaminoisoxazolones substituted on nitrogen with nitropyridine (12a-12e) in the presence of triethylamine provides imidazo heterocycles (13a-13e) which are suitable synthetic intermediates for a series of new planar polycyclic heterocycles that could be expected to have pharmaceutical applications. ${ }^{9,10}$ In addition, the formation of the isoxazolo[3,2-b]quinazoline $\mathbf{1 4}$ opens up intriguing possibilities of carbenoid derived products of novel structure.

\section{Experimental Section}

General Procedures. Freshly distilled solvents were used throughout, and anhydrous solvents were dried according to Perrin and Armarego. ${ }^{11}{ }^{1} \mathrm{H}(400 \mathrm{MHz})$ and ${ }^{13} \mathrm{C}(75 \mathrm{MHz}) \mathrm{NMR}$ measurements were recorded on a Brucker 400 spectrometer in deuteriochloroform with tetramethylsilane as internal standard, unless otherwise stated. Infrared spectra were recorded on a Thermonicolet (Nexus670) FT-infrared spectrometer, using sodium chloride cells, measured as Nujol mulls or films. Mass spectra were recorded on a Varian Matt 311 spectrometer and relative abundance of fragments is quoted in parentheses after the $\mathrm{m} / \mathrm{z}$ values. Melting points were determined on a Philip Harris C4954718 apparatus and are uncorrected. Microanalyses were performed on a Carlo-Erba Analyzer 1104 at the University of Giessen, Germany.

Diethyl (3-bromophenyl)thiocarbamoylmalonate (10a). In a $100 \mathrm{~mL}$ round-bottomed flask, absolute ethanol $(50 \mathrm{~mL})$ was reacted with sodium $(2.9 \mathrm{~g}, 0.126 \mathrm{~mol})$ and, after cooling to room temperature, diethylmalonate $(20 \mathrm{~g}, 18.95 \mathrm{ml}, 0.126 \mathrm{~mol})$ was added. The reaction mixture was stirred at room temperature for 15 minutes; 3-bromophenyl isothiocyanate (26.96 g, $0.126 \mathrm{~mol})$ was added and the stirring was continued for a further $6 \mathrm{~h}$, during which time a yellow-white precipitate of sodium diethyl (3-bromophenyl)thiocarbamoylmalonate salt was formed. The salt was collected and washed with light petroleum ether (b.p. $\left.30-60{ }^{\circ} \mathrm{C}\right)(3 \times 50 \mathrm{~mL})$ to give yellow crystals m.p. $157-158^{\circ} \mathrm{C}(34.22 \mathrm{~g}, 70 \%)$. The pure salt was dissolved in water $(40-50 \mathrm{~mL})$ and neutralized with dropwise addition of $\mathrm{HCl}(10 \%)$ to maintain the $\mathrm{pH}$ at 7 . The product was extracted with chloroform and the extract was washed with water $(3 \times 50 \mathrm{~mL})$ and dried over anhydrous $\mathrm{Na}_{2} \mathrm{SO}_{4}$. Removal of solvent gave (10a) as a yellow oil $(25.5 \mathrm{~g}, 68 \%)$. ${ }^{1} \mathrm{H}$ NMR $\left(\mathrm{CDCl}_{3}\right) \delta 1.29(\mathrm{t}, J=7.1 \mathrm{~Hz}, 3 \mathrm{H}), 4.27(\mathrm{q}, J=7.1 \mathrm{~Hz}, 2 \mathrm{H}), 5.06(\mathrm{~s}, 1 \mathrm{H}), 7.22(\mathrm{t}, J=8.06,1 \mathrm{H}), 7.36$ (bd, $J=7.95 \mathrm{~Hz}, 1 \mathrm{H}), 8.67$ (bd, $J=8.06 \mathrm{~Hz}, 1 \mathrm{H}), 8.05$ (t, $J=1.9 \mathrm{~Hz}, 1 \mathrm{H}), 10.83$ (bs, NH, 1H). FTIR $v_{\max } 3296,1747,1590,1541,1476,1394,1168,1023,680,784 \mathrm{~cm}^{-1}$.

Diethyl (3-methylphenyl)thiocarbamoylmalonate (10b). This compound was prepared as described above, using 3-methylphenyl isothiocyanate $(1.3 \mathrm{~g}, 8.7 \mathrm{mmol})$ and stirring for a further $1 \mathrm{~h}$ after addition of the isothiocyanate to diethylmalonate salt to give diethyl(3methylphenyl)thiocarbamoyl malonate $(1.924,71 \%)$ as a pale yellow solid, m.p.53-54 ${ }^{\circ} \mathrm{C} .{ }^{1} \mathrm{H}$ 
$\operatorname{NMR}\left(\mathrm{CDCl}_{3}\right) \delta 1.33(\mathrm{t}, \mathrm{J}=7.2 \mathrm{~Hz}, 6 \mathrm{H}), 2.38(\mathrm{~s}, 3 \mathrm{H}), 4.29(\mathrm{q}, \mathrm{J}=7.2 \mathrm{~Hz}, 2 \mathrm{H}), 4.30(\mathrm{q}, \mathrm{J}=7.2 \mathrm{~Hz}$, 2H), $5.09(\mathrm{~s}, 1 \mathrm{H}), 7.09(\mathrm{t}, \mathrm{J}=7.8 \mathrm{~Hz}, 1 \mathrm{H}), 7.55(\mathrm{bs}, 1 \mathrm{H}), 7.61(\mathrm{bd}, \mathrm{J}=8 \mathrm{~Hz}, 1 \mathrm{H}), 10.75(\mathrm{~s}$, exchanged by $\mathrm{D}_{2} \mathrm{O}$ addition, $\left.\mathrm{NH}, 1 \mathrm{H}\right)$. FT-IR $v_{\max } 3252,1752,1731,1592,1556,1422,1296$, $1148,1027,858,798,717 \mathrm{~cm}^{-1}$.

Diethyl (4-nitrophenyl)thiocarbamoylmalonate (10c). This compound was prepared as above, using 4-nitrophenylisothiocyanate $(0.5 \mathrm{~g}, 3.8 \mathrm{mmol})$ and stirring for a further $1 \mathrm{~h}$ after addition of the isothiocyanate to diethylmalonate salt to give diethyl (3methylphenyl)thiocarbamoylmalonate $(1.1 \mathrm{~g}, 97 \%)$ as a yellow solid, m.p.85-87 $\mathrm{C}$ (lit, $\left.87-90{ }^{\circ} \mathrm{C}\right) .^{5}$ Diethyl (3-ethoxycarbonylphenyl)thiocarbamoylmalonate (10d). This compound was prepared as described above, using 3-ethoxycarbonylphenyl isothiocyanate (26.08 g , $0.126 \mathrm{~mol})$ and stirring for a further $1 \mathrm{~h}$ after addition of the isothiocyanate, giving diethyl (3ethoxycarbonylphenyl) thiocarbamoylmalonate $(41.6 \mathrm{~g}, 90 \%)$ as a yellow oil. ${ }^{1} \mathrm{H}$ NMR $\delta(\mathrm{ppm})$ $1.31(\mathrm{t}, J=7.1 \mathrm{~Hz}, 6 \mathrm{H}), 1.38(\mathrm{t}, J=7.1 \mathrm{~Hz}, 3 \mathrm{H}), 4.29$ (q, $J=7.1 \mathrm{~Hz}, 2 \mathrm{H}), 4.30(\mathrm{q}, J=7.1 \mathrm{~Hz}, 2 \mathrm{H})$, $4.37(\mathrm{q}, J=7.1 \mathrm{~Hz}, 2 \mathrm{H}), 5.1(\mathrm{~s}, 1 \mathrm{H}), 7.48(\mathrm{t}, J=7.9 \mathrm{~Hz}, 1 \mathrm{H}), 7.92\left(\mathrm{dt}, J_{1}=7.8 \mathrm{~Hz}, J_{2}=1.7 \mathrm{~Hz}, 1 \mathrm{H}\right)$, $8.09\left(\mathrm{dt}, J_{1}=7.3 \mathrm{~Hz}, J_{2}=1.2 \mathrm{~Hz}, 1 \mathrm{H}\right), 8.33(\mathrm{t}, J=1.6 \mathrm{~Hz}, 1 \mathrm{H}), 10.90\left(\mathrm{~s}\right.$, exchanged by $\mathrm{D}_{2} \mathrm{O}$ addition , 1H, NH). ${ }^{13} \mathrm{C}$ NMR (ppm) 13.39, 13.78, 60.74, 62.56, 66.74, 123.93, 127.22, $127.39,128.40,130.77,138.22,165.07,165.16,188.10$. FT-IR $v_{\max } 3296,2983,1747,1721$, $1592,1445,1401,1309,1287,1217,1105,1025,758 \mathrm{~cm}^{-1}$.

Diethyl (4-ethoxycarbonylphenyl)thiocarbamoylmalonate (10e).This compound was prepared as described above, using 4-ethoxycarbonylphenyl isothiocyanate (26.08 g, $0.126 \mathrm{~mol})$ and stirring for a further $2 \mathrm{~h}$ after addition of the isothiocyanate, giving diethyl (4ethoxycarbonylphenyl) thiocarbamoylmalonate $\left(40.7 \mathrm{~g}, 88 \%\right.$ ) as a yellow oil. ${ }^{1} \mathrm{H}$ NMR $\delta$ (ppm) $1.29(\mathrm{t}, J=7.3 \mathrm{~Hz}, 6 \mathrm{H}), 1.37(\mathrm{t}, J=7.1 \mathrm{~Hz}, 3 \mathrm{H}), 4.27(\mathrm{q}, J=7.3 \mathrm{~Hz}, 2 \mathrm{H}), 4.28$ (q, $J=7.3 \mathrm{~Hz}, 2 \mathrm{H})$, 4.35 (q, $J=7.1 \mathrm{~Hz}, 2 \mathrm{H}), 5.08(\mathrm{~s}, 1 \mathrm{H}), 7.96(\mathrm{~d}, J=8.7 \mathrm{~Hz}, 2 \mathrm{H}), 8.05$ (d, J=8.7 Hz, 2H), 11.02 (s, exchanged by $\mathrm{D}_{2} \mathrm{O}$ addition, $\left.1 \mathrm{H}, \mathrm{NH}\right) .{ }^{13} \mathrm{C}$ NMR $\delta(\mathrm{ppm}) 13.33,13.74,60.47,62.50,67.02$, $121.69,127.75,129.74,141.85,164.99,165.04,187.69$. FT-IR $v_{\max } 3296,2983,1751,1720$, $1606,1547,1391,1277,1177,1108,1021,857,772 \mathrm{~cm}^{-1}$.

Diethyl (2-ethoxycarbonylphenyl)thiocarbamoylmalonate (10f). This compound was prepared as described above, using 2-ethoxycarbonylphenyl isothiocyanate (26.08 g, $0.126 \mathrm{~mol})$ and stirring for a further $2 \mathrm{~h}$ after addition of the isothiocyanate, giving diethyl (2ethoxycarbonylphenyl) thiocarbamoylmalonate $(43 \mathrm{~g}, 93 \%)$ as a yellow oil. ${ }^{1} \mathrm{H}$ NMR $(60 \mathrm{MHz}$, $\left.\mathrm{CDCl}_{3}\right): \delta(\mathrm{ppm}) 1.21(\mathrm{t}, J=7 \mathrm{~Hz}, 3 \mathrm{H}), 1.35(\mathrm{t}, J=7 \mathrm{~Hz}, 6 \mathrm{H}), 4.12(\mathrm{q}, J=7 \mathrm{~Hz}, 4 \mathrm{H}), 4.24(\mathrm{q}, J=$ $7 \mathrm{~Hz}, 2 \mathrm{H}), 4.93(\mathrm{~s}, 1 \mathrm{H}), 6.94(\mathrm{t}, J=7.5 \mathrm{~Hz}, 1 \mathrm{H}), 7.25$ (bt, $J=7 \mathrm{~Hz}, 1 \mathrm{H}), 7.73$ (bd, $J=7 \mathrm{~Hz}, 1 \mathrm{H}), 8.76$ (bd, $J=7.5 \mathrm{~Hz}, 1 \mathrm{H}), 12.44$ (bs, NH, 1H). FT-IR $\cup_{\max } 3252,2982,1735,1678,1620,1529,1488$, $1451,1402,1388,1302,1267,1200,1096,1023,758 \mathrm{~cm}^{-1}$.

Ethyl 3-(3-bromophenyl)amino-5-oxo-2,5-dihydroisoxazol-4-carboxylate (11a). To a solution of hydroxylamine hydrochloride $(2 \mathrm{~g}, 28.8 \mathrm{mmol})$ in water $(8 \mathrm{~mL})$, potassium bicarbonate $(2.8 \mathrm{~g}, 28 \mathrm{mmol})$ was added slowly. Ethanol $(32 \mathrm{~mL})$ was added and the resulting potassium chloride was filtered off. 
Diethyl (3-bromophenyl) thiocarbamoylmalonate $(3.4 \mathrm{~g}, 9 \mathrm{mmol})$ was added to the filtrate and refluxed for $24 \mathrm{~h}$. The reaction mixture was acidified with dilute hydrochloric acid and the white precipitate was collected by vacuum filtration. The white solid was recrystallized from ethanol to give the desired product $(2.51 \mathrm{~g}, 85 \%)$ as colourless crystals m.p. $100-102{ }^{\circ} \mathrm{C}$. Anal. Calc for $\mathrm{C}_{12} \mathrm{H}_{11} \mathrm{BrN}_{2} \mathrm{O}_{4} . \mathrm{H}_{2} \mathrm{~S}: \mathrm{C}, 41.73 ; \mathrm{H}, 3.19 ; \mathrm{N}, 8.11 \%$; found: C, 41.87; H, 3.22; N, 8.18\%. ${ }^{1} \mathrm{H}$ NMR $\left(\mathrm{D}_{6}-\mathrm{DMSO}+\mathrm{CDCl}_{3}\right) \delta 1.38(\mathrm{t}, J=7.1 \mathrm{~Hz}, 3 \mathrm{H}), 4.37(\mathrm{q}, J=7.1 \mathrm{~Hz}, 2 \mathrm{H}), 6.15(\mathrm{bs}, \mathrm{NH}$, $1 \mathrm{H}), 7.23\left(\mathrm{dt}, J_{1}=8.2 \mathrm{~Hz}, J_{2}=1.8 \mathrm{~Hz}, 1 \mathrm{H}\right), 7.28(\mathrm{t}, J=7.8 \mathrm{~Hz}, 1 \mathrm{H}), 7.33\left(\mathrm{dt}, J_{1}=7.8 \mathrm{~Hz}, J_{2}=1.6 \mathrm{~Hz}\right.$, $1 \mathrm{H}), 7.51(\mathrm{t}, J=1.8 \mathrm{~Hz}, 1 \mathrm{H}), 9.38(\mathrm{bs}, \mathrm{NH}, 1 \mathrm{H}),{ }^{13} \mathrm{C} \mathrm{NMR}\left(\mathrm{D}_{6}-\mathrm{DMSO}+\mathrm{CDCl}_{3}\right) \delta(\mathrm{ppm}), 14.49$, $60.26,75.21,119.80,122.92,123.82,128.24,130.95,137.58,162.85,165.37,166.72$. FT-IR $v_{\max } 3512,3297,1710,1701,1590,1478,1413,1323,1203,1116,1011,794,734 \mathrm{~cm}^{-1} ; \mathrm{MS} \mathrm{m} / \mathrm{z}$ (\%) 328( $\left.\mathrm{M}^{+}, 66 \%\right), 326\left(\mathrm{M}^{+}, 68 \%\right), 282(81), 280(70), 201(26), 197(16), 171(26), 157(36), 91(31)$, 90(32), 76(21), 63(24), 45(19), 44(100), 40(47), 36(23) and HRMS m/z 325.99021 $\left(\mathrm{C}_{12} \mathrm{H}_{11} \mathrm{BrN}_{2} \mathrm{O}_{4}\right.$ requires 325.99022).

Ethyl 3-(3-methylphenyl)amino-5-oxo-2,5-dihydroisoxazol-4-carboxylate (11b). The compound was prepared as described above using diethyl(3-methylphenyl) thiocarbamoylmalonate $(1.1 \mathrm{~g}, 3.5 \mathrm{mmol})$ and refluxing for $24 \mathrm{~h}$ to give the desired product as colourless crystals $(0.7 \mathrm{~g}, 75 \%)$, m.p. $109-111{ }^{\circ} \mathrm{C} .{ }^{1} \mathrm{H}$ NMR(D $\left(\mathrm{D}_{6}-\mathrm{DMSO}+\mathrm{CDCl}_{3}\right) \delta 1.38$ (t, $J=$ $7.1 \mathrm{~Hz}, 3 \mathrm{H}), 2.37$ (s,3H), 4.35 (q, $J=7.1 \mathrm{~Hz}, 2 \mathrm{H}), 7.02$ (bd, $J=7.6 \mathrm{~Hz}, 1 \mathrm{H}), 7.07$ (bd, $J=7.9 \mathrm{~Hz}$, $1 \mathrm{H}), 7.1(\mathrm{bs}, 1 \mathrm{H}), 7.27(\mathrm{t}, J=7.8 \mathrm{~Hz}, 1 \mathrm{H}), 9.31(\mathrm{~s}, 1 \mathrm{H}, \mathrm{NH}) .{ }^{13} \mathrm{C}$ NMR $\left(\mathrm{D}_{6}-\mathrm{DMSO}+\mathrm{CDCl}_{3}\right)$ $\delta(\mathrm{ppm}) 14.53,21.34,60.17,74.82,118.19,121.82,126.42,129.47,135.90,139.75,163.35$, 165.64, 166.66. FT-IR $v_{\max } 3519$, 3316, 1710, 1678, 1578, 1324, 1226, 1169, 1113, 1000, 795, $725 \mathrm{~cm}^{-1}$.

Ethyl 3-(4-nitrophenyl)amino-5-oxo-2,5-dihydroisoxazol-4-carboxylate (11c). This compound was prepared as described above using diethyl (4-nitrophenyl)thiocarbamoylmalonate $(1.1 \mathrm{~g}, 3.23 \mathrm{mmol})$ and refluxing for 24 hours to give the desired product $(0.62 \mathrm{~g}, 65 \%)$ as a yellow solid, m.p. $158-160^{\circ} \mathrm{C}\left(\right.$ lit. $\left.161-163^{\circ} \mathrm{C}\right) .^{5}$

${ }^{1} \mathrm{HNMR}\left(\mathrm{D}_{6}-\mathrm{DMSO}+\mathrm{CDCl}_{3}\right) \delta 1.30(\mathrm{t}, J=7.1 \mathrm{~Hz}, 3 \mathrm{H}), 4.36(\mathrm{q}, J=7.1 \mathrm{~Hz}, 2 \mathrm{H}), 6.32(\mathrm{bs}, \mathrm{NH}$, $1 \mathrm{H}), 7.51(\mathrm{~d}, \mathrm{~J}=9.1 \mathrm{~Hz}, 2 \mathrm{H}), 8.23(\mathrm{~d}, \mathrm{~J}=9.1,2 \mathrm{H}), 9.53(\mathrm{~s}, \mathrm{NH}, 1 \mathrm{H}) .{ }^{13} \mathrm{C} \mathrm{NMR}_{\left(\mathrm{D}^{-}\right.}$ $\left.\mathrm{DMSO}+\mathrm{CDCl}_{3}\right) \delta 14.43,60.37,76.45,118.82,125.33,142.68,143.53,161.35,164.74,168.48$.

FT-IR $v_{\max } 3470,1755,1723,1681,1632,1582,1518,1498,1418,1345,1211,1117,1021$, $857,790,745 \mathrm{~cm}^{-1}$.

Ethyl 3-(3-ethoxycarbonylphenyl)amino-5-oxo-2,5-dihydroisoxazole-4-carboxylate (11d). This compound was prepared as described above using diethyl (3-ethoxycarbonylphenyl) thiocarbamoylmalonate $(12.48 \mathrm{~g}, 34 \mathrm{mmol})$ and refluxing for 48 hours to give the desired product $\left(4.35 \mathrm{~g}, 40 \%\right.$ ) as white needles, m.p. $185-187^{\circ} \mathrm{C}$. Anal. Calc for $\mathrm{C}_{15} \mathrm{H}_{16} \mathrm{~N}_{2} \mathrm{O}_{6}: \mathrm{C}, 56.25$; $\mathrm{H}, 5.00$; N, 8.75\%; found: C, 55.98; H, 4.93; N, 9.00\%. ${ }^{1} \mathrm{H}$ NMR (D $\left.-\mathrm{DMSO}\right) \delta(\mathrm{ppm}) 1.23(\mathrm{t}, J=$ $7.05 \mathrm{~Hz}, 3 \mathrm{H}), 1.32$ (t, $J=7.1 \mathrm{~Hz}, 3 \mathrm{H}), 4.15$ (q, $J=7.05 \mathrm{~Hz}, 2 \mathrm{H}), 4.31$ (q, $J=7.1 \mathrm{~Hz}, 2 \mathrm{H}), 4.40$ (bs , exchanged by $\mathrm{D}_{2} \mathrm{O}$ addition, $\left.1 \mathrm{H}, \mathrm{NH}\right), 7.44(\mathrm{t}, J=7.9 \mathrm{~Hz}, 1 \mathrm{H}), 7.60(\mathrm{bd}, J=7.7 \mathrm{~Hz}, 1 \mathrm{H}), 7.63$ $\left(\mathrm{dt}, J_{1}=8.1 \mathrm{~Hz}, J_{2}=1.1 \mathrm{~Hz}, 1 \mathrm{H}\right), 8.03(\mathrm{bs}, 1 \mathrm{H}), 9.06\left(\mathrm{~s}\right.$, exchanged by $\mathrm{D}_{2} \mathrm{O}$ addition, $\left.1 \mathrm{H}, \mathrm{NH}\right) .{ }^{13} \mathrm{C}$

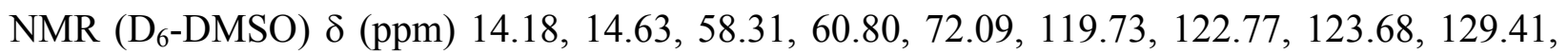


130.77, 139.45, 161.47, 164.90, 165.63, 169.20. FT-IR $v_{\max } 3403,2986,1697,1694,1611,1555$, $1492,1455,1369,1293,1216,1111,1087,1026,784,747 \mathrm{~cm}^{-1}$.

Ethyl 3-(4-ethoxycarbonylphenyl)amino-5-oxo-2,5-dihydroisoxazole-4-carboxylate (11e). This compound was prepared as described above using diethyl (4-ethoxycarbonylphenyl) thiocarbamoylmalonate $(12.48 \mathrm{~g}, 34 \mathrm{mmol})$ and refluxing for 24 hours to give the desired product $(7.07 \mathrm{~g}, 65 \%)$ as white needles, m.p. 126-129 ${ }^{\circ} \mathrm{C}$. Anal. Calc for $\mathrm{C}_{15} \mathrm{H}_{16} \mathrm{~N}_{2} \mathrm{O}_{6}: \mathrm{C}, 56.25$; $\mathrm{H}, 5.00 ; \mathrm{N}, 8.75 \%$; found: $\mathrm{C}, 55.88 ; \mathrm{H}, 4.92 ; \mathrm{N}, 8.92 \% .{ }^{1} \mathrm{H} \mathrm{NMR}\left(\mathrm{CDCl}_{3}+\mathrm{D}_{6}\right.$-DMSO) $\delta$ (ppm) $1.38(\mathrm{t}, J=7.1 \mathrm{~Hz}, 3 \mathrm{H}), 1.40(\mathrm{t}, J=7.1 \mathrm{~Hz}, 3 \mathrm{H}), 4.35(\mathrm{q}, J=7.1 \mathrm{~Hz}, 4 \mathrm{H}), 7.34(\mathrm{~d}, J=8.7 \mathrm{~Hz}, 2 \mathrm{H})$, 7.55 (bs, exchanged by $\mathrm{D}_{2} \mathrm{O}$ addition, $\left.1 \mathrm{H}, \mathrm{NH}\right), 8.04(\mathrm{~d}, J=8.7 \mathrm{~Hz}, 2 \mathrm{H}), 9.58$ (s, exchanged by $\mathrm{D}_{2} \mathrm{O}$ addition, $\left.1 \mathrm{H}, \mathrm{NH}\right) .{ }^{13} \mathrm{C} \mathrm{NMR}\left(\mathrm{CDCl}_{3}+\mathrm{D}_{6}\right.$-DMSO) $\delta(\mathrm{ppm}) 14.29,14.47,60.35,60.98$, $75.69,119.39,126.40,131.13,140.52,162.49,165.33,165.62,166.92$. FT-IR $U_{\max } 3278,2984$, $2761,1716,1683,1601,1575,1471,1415,1369,1329,1278,1184,1104,1022,798,769 \mathrm{~cm}^{-1}$

Ethyl 3-(3-bromophenyl)amino-2-(5-nitropyrid-2-yl)-5-oxo-2,5-dihydroisoxazol-4-carboxylate (12a). A mixture of 2-chloro-5-nitropyridine (58mg, $0.367 \mathrm{mmol}$ ) and ethyl 3-(3bromophenyl)amino-5-oxo-2,5-dihydroisoxazol-4-carboxylate $(0.12 \mathrm{~g}, 0.367 \mathrm{mmol})$ was heated neat under an atmosphere of nitrogen in an oil bath at $130{ }^{\circ} \mathrm{C}$ for $1.5 \mathrm{~h}$. The residue was recrystallized from ethanol to give the desired isoxazolone as yellow crystals $(75 \mathrm{mg}, 46 \%)$, m.p.207-209 ${ }^{\circ}$ C. Anal. Calc for $\mathrm{C}_{17} \mathrm{H}_{13} \mathrm{BrN}_{4} \mathrm{O}_{6}$ : C, 45.43, H, $2.89 \mathrm{~N}, 12.47 \%$; found: C, 45.38; $\mathrm{H}$, 2.57; N, 12.26\%. ${ }^{1} \mathrm{HNMR}\left(\mathrm{D}_{6}-\mathrm{DMSO}+\mathrm{CDCl}_{3}\right) \delta 1.14(\mathrm{t}, J=7.1 \mathrm{~Hz}, 3 \mathrm{H}), 4.04(\mathrm{q}, J=7.1 \mathrm{~Hz}, 2 \mathrm{H})$, $7.20(\mathrm{t}, J=2.9 \mathrm{~Hz}, 1 \mathrm{H}), 7.23\left(\mathrm{dt}, J_{1}=8.0 \mathrm{~Hz}, J_{2}=1.9 \mathrm{~Hz}, 1 \mathrm{H}\right), 7.29\left(\mathrm{dt}, J_{1}=7.3 \mathrm{~Hz}, J_{2}=1.9 \mathrm{~Hz}, 1 \mathrm{H}\right)$, $7.45(\mathrm{t}, J=1.9 \mathrm{~Hz}, 1 \mathrm{H}), 7.62(\mathrm{~d}, J=9.1 \mathrm{~Hz}, 1 \mathrm{H}), 8.69\left(\mathrm{dd}, J_{1}=9.1 \mathrm{~Hz}, J_{2}=2.7 \mathrm{~Hz}, 1 \mathrm{H}\right), 9.08(\mathrm{~d}, J=$ $2.7 \mathrm{~Hz}, 1 \mathrm{H}), 10.72(\mathrm{~s}, \mathrm{NH}, 1 \mathrm{H}) .{ }^{13} \mathrm{C} \mathrm{NMR}\left(\mathrm{D}_{6}-\mathrm{DMSO}+\mathrm{CDCl}_{3}\right) \delta 13.99,60.13,78.89,114.46$, $121.27,121.85,125.85,128.61,130.33,134.98,139.29,141.35,143.60,153.62,158.82$, 161.72,162.83. FT-IR $v_{\max } 3153,1778,1698,1596,1552,1517,1333,1200,1122,969,756$

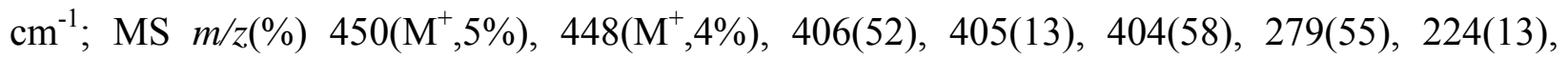
157(13), 155(13), 78(13), 77(14), 44(100), 40(52) and HRMS $448.00184\left(\mathrm{C}_{17} \mathrm{H}_{13} \mathrm{BrN}_{4} \mathrm{O}_{6}\right.$ requires 448.00185$)$.

Ethyl (3-methyloxycarbonylphenyl)amino-2-(5-nitropyrid-2-yl)-5-oxo-2,5-dihydroisoxazol4-carboxylate (12b). This compound was prepared as described above using the corresponding isoxazolone $(180 \mathrm{mg}, 0.69 \mathrm{mmol})$ and 2-chloro-5-nitropyridine $(109 \mathrm{mg}, 0.69 \mathrm{mmol})$ to give the desired product as yellow needles $(0.13 \mathrm{~g}, 46 \%)$ after recrystalization from ethanol, m.p.160$163{ }^{\circ} \mathrm{C}$. Anal. Calc for $\mathrm{C}_{18} \mathrm{H}_{16} \mathrm{~N}_{4} \mathrm{O}_{6}: \mathrm{C}, 56.25, \mathrm{H}, 4.16, \mathrm{~N}, 14.58 \%$; found: $\mathrm{C}, 56.10 ; \mathrm{H}, 3.86$; N, 14.28\%. ${ }^{1} \mathrm{HNMR}\left(\mathrm{CDCl}_{3}\right) \delta 1.26(\mathrm{t}, J=7.1 \mathrm{~Hz}, 3 \mathrm{H}), 2.28(\mathrm{~s}, 3 \mathrm{H}), 4.22(\mathrm{q}, J=7.1 \mathrm{~Hz}, 2 \mathrm{H}), 6.62-6.97$ $(\mathrm{m}, 3 \mathrm{H}), 7.13(\mathrm{t}, J=7.7 \mathrm{~Hz}, 1 \mathrm{H}), 7.54(\mathrm{~d}, J=9.1 \mathrm{~Hz}, 1 \mathrm{H}), 8.54\left(\mathrm{dd}, J_{1}=9.1 \mathrm{~Hz}, J_{2}=2.6 \mathrm{~Hz}, 1 \mathrm{H}\right), 8.91$ $(\mathrm{d}, J=2.6 \mathrm{~Hz}, 1 \mathrm{H}), 10.35(\mathrm{~s}, \mathrm{NH}, 1 \mathrm{H}) .{ }^{13} \mathrm{C} \mathrm{NMR}\left(\mathrm{CDCl}_{3}\right) \delta 14.25,21.27,60.98,78.86,114.82$, $118.99,122.64,127.21,129.14,134.34,137.41,139.53,141.45,143.54,153.87,160.13,163.15$, 163.64. FT-IR $v_{\max } 3176,1782,1702,1567,1511,1332,1206,1173,1118,968,756 \mathrm{~cm}^{-1}$; MS $\mathrm{m} / \mathrm{z}(\%)$ 384( $\left.\mathrm{M}^{+}, 20 \%\right), 341(24), 340(100), 248(31), 230(28), 158(45), 107(20), 91(70), 65(23)$, 44(84), 40(36) and HRMS $384.10698\left(\mathrm{C}_{18} \mathrm{H}_{16} \mathrm{~N}_{4} \mathrm{O}_{6}\right.$ requires 384.10699). 
Ethyl 3-(4-nitrophenyl)amino-2-(5-nitropyrid-2-yl)-5-oxo-2,5-dihydroisoxazol-4-carboxylate (12c). This compound was prepared as described for above using the corresponding isoxazolone (100mg, $0.34 \mathrm{mmol})$ and 2-chloro-5-nitropyridine $(63 \mathrm{mg}, 0.4 \mathrm{mmol})$ to give the desired product as cream solid $(48.2 \mathrm{mg}, 35 \%)$ after recrystalization from ethanol, m.p.223-226 $6^{\circ} \mathrm{C}$ (lit,225-228 $\left.{ }^{\circ} \mathrm{C}\right) .^{5}$ Anal. Calc for $\mathrm{C}_{17} \mathrm{H}_{13} \mathrm{~N}_{5} \mathrm{O}_{8}$ : C, 49.15; $\mathrm{H}, 3.13 ; \mathrm{N}, 16.86 \%$; found: $\mathrm{C}, 48.83 ; \mathrm{H}, 2.72 ; \mathrm{N}, 16.59$. ${ }^{1} \mathrm{HNMR}\left(\mathrm{D}_{6}-\mathrm{DMSO}+\mathrm{CDCl}_{3}\right) \delta 1.17(\mathrm{t}, J=7.1 \mathrm{~Hz}, 3 \mathrm{H}), 4.12(\mathrm{q}, J=7.1 \mathrm{~Hz}, 2 \mathrm{H}), 7.47(\mathrm{~d}, J=9.1 \mathrm{~Hz}$, 2H), $7.69(\mathrm{~d}, J=9.1 \mathrm{~Hz}, 1 \mathrm{H}), 8.14(\mathrm{~d}, J=9.1 \mathrm{~Hz}, 2 \mathrm{H}), 8.71\left(\mathrm{dd}, J_{1}=9.1 \mathrm{~Hz}, J_{2}=2.7 \mathrm{~Hz}, 1 \mathrm{H}\right), 9.02(\mathrm{~d}$, $J=2.7 \mathrm{~Hz}, 1 \mathrm{H}), 10.93(\mathrm{~s}, \mathrm{NH}, 1 \mathrm{H}) .{ }^{13} \mathrm{C} \mathrm{NMR}\left(\mathrm{D}_{6}-\mathrm{DMSO}+\mathrm{CDCl}_{3}\right) \delta 14.05,60.21,80.66,114.44$, $121.61,124.45,135.11,141.52,143.53,143.87,144.43,153.34,158.29,161.61,162.43$. FT-IR $v_{\max } 3114,1782,1687,1602,1553,1428,1337,1261,1202,1122,1082,1011,960,858,552$ $\mathrm{cm}^{-1}$; MS m/z(\%) 415(M+12\%), 371(78), 369(11), 325(13), 300(11), 279(29), 189(18), 149(12), 70(11), 44(100). 40(38) and HRMS $415.07641\left(\mathrm{C}_{17} \mathrm{H}_{13} \mathrm{~N}_{5} \mathrm{O}_{8}\right.$ requires 415.07641).

Ethyl 3-(3-ethoxycarbonylphenyl)amino-2-(5-nitropyrid-2-yl)-5-oxo-2,5-dihydroisoxazole4-carboxylate (12d). This compound was prepared as described above using the corresponding isoxazolone (96 mg, $0.3 \mathrm{mmol}$ ) and 2-chloro-5-nitropyridine (48.5 $\mathrm{mg}, 0.3 \mathrm{mmol})$ to give the desired product $(60 \mathrm{mg}, 45 \%)$ as cream needles, m.p. 193-195 ${ }^{\circ} \mathrm{C}$ (decomposed). Anal. Calc for $\mathrm{C}_{20} \mathrm{H}_{18} \mathrm{~N}_{4} \mathrm{O}_{8}$ : C, 54.29; H, 4.07; N, 12.67\%; found: C, 54.08; H, 3.80; N, $13.00 \%$. ${ }^{1} \mathrm{H}$ NMR $\delta$ (ppm) $1.26(\mathrm{t}, J=7.1 \mathrm{~Hz}, 3 \mathrm{H}), 1.39$ (t, $J=7.1 \mathrm{~Hz}, 3 \mathrm{H}), 4.23$ (q, $J=7.1 \mathrm{~Hz}, 2 \mathrm{H}), 4.36$ (q, $J=7.1 \mathrm{~Hz}$, 2H), 7.33 (bs, $1 \mathrm{H}, 7.37$ (t, $J=7.8 \mathrm{~Hz}, 1 \mathrm{H}), 7.59$ (d, $J=9.1 \mathrm{~Hz}, 1 \mathrm{H}), 7.82(\mathrm{bd}, J=7.0 \mathrm{~Hz}, 2 \mathrm{H}), 8.57$ $\left(\mathrm{dd}, J_{1}=9.1 \mathrm{~Hz}, J_{2}=2.6 \mathrm{~Hz}, 1 \mathrm{H}\right), 8.90(\mathrm{~d}, J=2.6 \mathrm{~Hz}, 1 \mathrm{H}), 10.54\left(\mathrm{~s}\right.$, exchanged by $\mathrm{D}_{2} \mathrm{O}$ addition, $1 \mathrm{H}, \mathrm{NH}) .{ }^{13} \mathrm{C}$ NMR $\delta$ (ppm) 14.27, 14.28, 61.11, 61.50, 79.33, 114.58, 122.83, 125.93, 127.25, 129.40, 131.83, 134.60, 137.94, 141.47, 143.49, 153.71, 159.88, 162.82, 163.51, 165.35. FT-IR $\mathrm{U}_{\max } 3371,3107,2958,1782,1712,1692,1567,1532,1451,1421,1337,1285,1204,1118$, $1028,971,859,757 \mathrm{~cm}^{-1}$.

Ethyl 3-(4-ethoxycarbonylphenyl)amino-2-(5-nitropyrid-2-yl)-5-oxo-2,5-dihydroisoxazole4-carboxylate (12e). This compound was prepared as described above using the corresponding isoxazolone (96 mg , $0.3 \mathrm{mmol}$ ) and 2-chloro-5-nitropyridine $(48.5 \mathrm{mg}, 0.3 \mathrm{mmol})$ to give the desired product (63 mg, 48\%) as white needles, m.p. 197-200 ${ }^{\circ} \mathrm{C}$. Anal. Calc for $\mathrm{C}_{20} \mathrm{H}_{18} \mathrm{~N}_{4} \mathrm{O}_{8}$ : C, 54.29; H,4.07; N,12.67\%; found: C,54.10; H,4.26; N,12.86\%. ${ }^{1} \mathrm{H}$ NMR $\delta$ (ppm) 1.29 (t, J= $7.1 \mathrm{~Hz}, 3 \mathrm{H}), 1.38(\mathrm{t}, J=7.1 \mathrm{~Hz}, 3 \mathrm{H}), 4.27(\mathrm{q}, J=7.1 \mathrm{~Hz}, 2 \mathrm{H}), 4.35$ (q, $J=7.1 \mathrm{~Hz}, 2 \mathrm{H}), 7.19$ (d, $J=$ $8.6 \mathrm{~Hz}, 2 \mathrm{H}), 7.59(\mathrm{~d}, J=9.1 \mathrm{~Hz}, 1 \mathrm{H}), 7.96(\mathrm{~d}, J=8.6 \mathrm{~Hz}, 2 \mathrm{H}), 8.58\left(\mathrm{dd}, J_{1}=9.1 \mathrm{~Hz}, J_{2}=2.6 \mathrm{~Hz}\right.$, $1 \mathrm{H}), 8.88(\mathrm{~d}, J=2.6 \mathrm{~Hz}, 1 \mathrm{H}), 10.54$ (s, exchanged by $\mathrm{D}_{2} \mathrm{O}$ addition, $\left.1 \mathrm{H}, \mathrm{NH}\right) .{ }^{13} \mathrm{C} \mathrm{NMR} \delta(\mathrm{ppm})$ $14.28,29.72,61.25,61.28,79.86,114.53,120.90,127.95,130.90,134.62,141.56,141.65$, $143.49,153.68,159.78,162.64,163.69,165.44$. FT IR $v_{\max } 3394,3154,2982,1780,1703,1602$, $1470,1425,1343,1278,1201,1177,1118,1021,859,757 \mathrm{~cm}^{-1}$.

Ethyl 2-(3-bromo phenyl)amino-6-nitroimidazo[1,2-a]pyridine-3-carboxylate (13a). The isoxazolone (12a) $(65 \mathrm{mg}, 0.145 \mathrm{mmol})$ and triethylamine $(0.13 \mathrm{ml})$ were refluxed in ethanol $(10 \mathrm{ml})$ for $12 \mathrm{~h}$. The reaction mixture was left to cool to room temperature and the resulting precipitate was collected to give (13a) as yellow needles (35mg, 60\%), m.p.161-163 ${ }^{\circ} \mathrm{C}$. Anal. Calc for $\mathrm{C}_{16} \mathrm{H}_{13} \mathrm{BrN}_{4} \mathrm{O}_{4}$ : C, 47.4, H, 3.2, N, 13.82\%; found: C, 47.40; H, 2.85; N, 13.58\%. 
${ }^{1} \mathrm{HNMR}\left(\mathrm{D}_{6}-\mathrm{DMSO}+\mathrm{CDCl}_{3}\right) \delta 1.55(\mathrm{t}, J=7.1 \mathrm{~Hz}, 3 \mathrm{H}), 4.57(\mathrm{q}, J=7.1 \mathrm{~Hz}, 2 \mathrm{H}), 7.18\left(\mathrm{bdd}, J_{1}=\right.$ $\left.8.0 \mathrm{~Hz}, J_{2}=1.6 \mathrm{~Hz}, 1 \mathrm{H}\right), 7.22(\mathrm{t}, J=7.8 \mathrm{~Hz}, 1 \mathrm{H}), 7.56\left(\mathrm{dt}, J_{1}=7.4 \mathrm{~Hz}, J_{2}=1.9 \mathrm{~Hz}, 1 \mathrm{H}\right), 7.58(\mathrm{~d}, J=$ $9.6 \mathrm{~Hz}, 1 \mathrm{H}), 8.06(\mathrm{bs}, 1 \mathrm{H}), 8.18\left(\mathrm{dd}, J_{1}=9.6 \mathrm{~Hz}, J_{2}=2.1 \mathrm{~Hz}, 1 \mathrm{H}\right), 8.95(\mathrm{bs}, 1 \mathrm{H}), 9.85(\mathrm{bs}, 1 \mathrm{H}) .{ }^{13} \mathrm{C}$ $\mathrm{NMR}\left(\mathrm{D}_{6}\right.$-DMSO$\left.+\mathrm{CDCl}_{3}\right) \delta 14.63,61.28,99.23,114.42,116.64,117.13,116.06,121.23,122.55$, $122.93,125.59,126.95,130.40,137.18,140.72,160.28$. FT-IR $v_{\max } 3306,1664,1590,1561$, 1457, 1407, 1342, 1308, 1212, 1134, 1106, 847, $763 \mathrm{~cm}^{-1} ; \mathrm{MS} \mathrm{m} / \mathrm{z}(\%) 406\left(\mathrm{M}^{+}, 89 \%\right)$, 404( $\left.\mathrm{M}^{+}, 86 \%\right), 279(100), 251(16), 233(14), 206(11), 184(12), 182(11), 102(12), 78(11), 77(11)$, 44(26), 40(50) and HRMS $404.01201\left(\mathrm{C}_{16} \mathrm{H}_{13} \mathrm{BrN}_{4} \mathrm{O}_{4}\right.$ requires 404.01202).

Ethyl 2-(3-methylphenyl)amino-6-nitroimidazo[1,2-a] pyridine-3-carboxylate (13b). The isoxazolone $(12 \mathrm{~b})(126 \mathrm{mg}, 0.37 \mathrm{mmol})$ and triethylamine $(0.13 \mathrm{ml})$ were refluxed in ethanol $(10 \mathrm{ml})$, for 3 hours. The reaction mixture was left to cool to room temperature and resulting precipitate was collected to give (13b) as a yellow solid (60mg, 54\%), m.p.167-169 ${ }^{\circ} \mathrm{C}$. Anal. Calc for $\mathrm{C}_{17} \mathrm{H}_{16} \mathrm{~N}_{4} \mathrm{O}_{4}$ : C, $60.00 \mathrm{H}, 4.70 ; \mathrm{N}, 16.47 \%$; found: C,59.58; H,4.32; N,16.00. ${ }^{1} \mathrm{HNMR}$ $\left(\mathrm{CDCl}_{3}\right) \delta 1.54(\mathrm{t}, J=7.1 \mathrm{~Hz}, 3 \mathrm{H}), 2.40(\mathrm{~s}, 3 \mathrm{H}), 4.56(\mathrm{q}, J=7.1 \mathrm{~Hz}, 2 \mathrm{H}), 6.90(\mathrm{~d}, J=7.4 \mathrm{~Hz}, 1 \mathrm{H})$, $7.26(\mathrm{t}, J=7.8 \mathrm{~Hz}, 1 \mathrm{H}), 7.50-7.55(\mathrm{~m}, 3 \mathrm{H}), 8.15(\mathrm{~d}, J=9.7,1 \mathrm{H}), 8.84(\mathrm{bs}, 1 \mathrm{H}), 9.94(\mathrm{bs}, 1 \mathrm{H}) .{ }^{13} \mathrm{C}$ $\mathrm{NMR}\left(\mathrm{CDCl}_{3}\right) \delta 14.59,21.62,61.05,98.75,113.94,115.74,119.20,122.20,123.98,126.79$, 129.01, 139.07, 139.22, 145.02, 146.84, 156.33, 167.64. FT-IR $v_{\max } 3403,1655,1613,1578$, 1551, 1493, 1344, 1308, 1216, 958, $767 \mathrm{~cm}^{-1}$; MS m/z(\%) 340(M+100\%),296(14), 294(40), 255(23), 248(30), 118(13), 91(25), 86(19), 44(11), 40(35) and HRMS $340.11715\left(\mathrm{C}_{17} \mathrm{H}_{16} \mathrm{~N}_{4} \mathrm{O}_{4}\right.$ requires 340.11716$)$.

Ethyl 2-(4-nitrophenyl)amino-6-nitroimidazo[1,2-a] pyridine-3-carboxylate (13c). The isoxazolone $(12 \mathrm{c})(100 \mathrm{mg}, 0.24 \mathrm{mmol})$ and triethylamine $(0.13 \mathrm{ml})$ were refluxed in ethanol $(10$ $\mathrm{ml})$ for 24 hours. The reaction mixture was left to cool to room temperature and the resulting precipitate was collected to give (13e) as a green solid (67mg, 72\%), m.p.259-261 ${ }^{\circ} \mathrm{C}$. Anal. Calc for $\mathrm{C}_{16} \mathrm{H}_{13} \mathrm{~N}_{5} \mathrm{O}_{6}$ : C, 51.75; H, 3.50; N, 18.86\%; found: C, 51.33; H, 3.12; N, 18.63\%. ${ }^{1} \mathrm{HNMR}$ $\left(\mathrm{D}_{6}-\mathrm{DMSO}+\mathrm{CDCl}_{3}\right) \delta 1.44(\mathrm{t}, J=7.1 \mathrm{~Hz}, 3 \mathrm{H}), 4.50(\mathrm{q}, J=7.1 \mathrm{~Hz}, 2 \mathrm{H}), 7.83(\mathrm{~d}, J=9.7 \mathrm{~Hz}, 1 \mathrm{H}), 8.08$ $(\mathrm{d}, J=9.2 \mathrm{~Hz}, 2 \mathrm{H}), 8.25(\mathrm{~d}, J=9.2 \mathrm{~Hz}, 2 \mathrm{H}), 8.28\left(\mathrm{dd}, J_{1}=9.7 \mathrm{~Hz}, J_{2}=2.4 \mathrm{~Hz}, 1 \mathrm{H}\right), 9.30(\mathrm{bs}, \mathrm{NH}, 1 \mathrm{H})$, 10.02 (bd, $J=1.9 \mathrm{~Hz}, 1 \mathrm{H})$. FT-IR $v_{\max } 3381,1664,1609,1575,1509,1475,1317,1273,1209$, 1102, 860, $749 \mathrm{~cm}^{-1}$; MS m/z (\%) 371( $\left.\mathrm{M}^{+}, 100\right), 325(12), 280(9), 279(35), 251(11), 205(12)$, 76(9), 44(16), 40(23) and HRMS $371.08658\left(\mathrm{C}_{16} \mathrm{H}_{13} \mathrm{~N}_{5} \mathrm{O}_{6}\right.$ requires 371.08659).

Ethyl 2-(3-ethoxycarbonylphenyl)amino-6-nitroimidazo[1,2-a] pyridine-3-carboxylate (13d). The isoxazolone (12d) (106 mg, $0.24 \mathrm{mmol})$ and triethylamine $(0.2 \mathrm{~mL})$ were refluxed in ethanol $(10 \mathrm{ml})$ for 3 hours. The reaction mixture was left to cool to room temperature and the resulting precipitate was collected to give (13d) (61 mg, 64\%) as yellow needles, m.p. $172-174^{\circ} \mathrm{C}$. Anal. Calc for $\mathrm{C}_{19} \mathrm{H}_{18} \mathrm{~N}_{4} \mathrm{O}_{6}$ : C, 58.28; H,4.52; N,14.07\%; found: C,58.54; H,4.48; N,14.45\%. ${ }^{1} \mathrm{H}$ NMR $\delta(\mathrm{ppm}) 1.43(\mathrm{t}, J=7.1 \mathrm{~Hz}, 3 \mathrm{H}), 1.56(\mathrm{t}, J=7.1 \mathrm{~Hz}, 3 \mathrm{H}), 4.41(\mathrm{q}, J=7.1 \mathrm{~Hz}, 2 \mathrm{H}), 4.58(\mathrm{q}, J=7.1 \mathrm{~Hz}$, $2 \mathrm{H}), 7.45(\mathrm{t}, J=7.9 \mathrm{~Hz}, 1 \mathrm{H}), 7.56(\mathrm{~d}, J=9.7 \mathrm{~Hz}, 1 \mathrm{H}), 7.74(\mathrm{bd}, J=7.7 \mathrm{~Hz}, 1 \mathrm{H}), 8.09\left(\mathrm{dd}, J_{1}=\right.$ $\left.8.03 \mathrm{~Hz}, J_{2}=1.3 \mathrm{~Hz}, 1 \mathrm{H}\right), 8.17\left(\mathrm{dd}, J_{1}=9.7 \mathrm{~Hz}, J_{2}=1.7 \mathrm{~Hz}, 1 \mathrm{H}\right), 8.26(\mathrm{bs}, 1 \mathrm{H}), 9.03$ (bs, exchanged by $\mathrm{D}_{2} \mathrm{O}$ addition, $\left.1 \mathrm{H}, \mathrm{NH}\right), 9.86(\mathrm{bs}, 1 \mathrm{H}) .{ }^{13} \mathrm{C} \mathrm{NMR} \delta(\mathrm{ppm}) 14.36,14.65,61.16,61.26,99.14$, $114.37,119.67,122.57,122.83,123.75,126.97,129.27,131.50,137.12,139.62,146.86,166.40$. 
FT-IR $v_{\max } 3403,3325,2923,1716,1675,1612,1576,1550,1490,1346,1315,1281,1230$, $1107,750 \mathrm{~cm}^{-1}$.

Ethyl 2-(4-ethoxycarbonylphenyl)amino-6-nitroimidazo[1,2-a] pyridine-3-carboxylate (13e). The isoxazolone $(12 \mathrm{e})(106 \mathrm{mg}, 0.24 \mathrm{mmol})$ and triethylamine $(0.2 \mathrm{~mL})$ were refluxed in ethanol $(10 \mathrm{ml})$ for 3 hours. The reaction mixture was left to cool to room temperature and the resulting precipitate was collected to give (13e) $(67 \mathrm{mg}, 70 \%)$ as bright yellow needles, m.p. $208-210^{\circ} \mathrm{C}$. Anal. Calc for $\mathrm{C}_{19} \mathrm{H}_{18} \mathrm{~N}_{4} \mathrm{O}_{6}$ : C, 58.28; H,4.52; N,14.07\%; found: C,58.01; H,4.33; N,14.27\%. ${ }^{1} \mathrm{H}$ $\operatorname{NMR} \delta(\mathrm{ppm}) 1.40(\mathrm{t}, J=7.1 \mathrm{~Hz}, 3 \mathrm{H}), 1.56(\mathrm{t}, J=7.1 \mathrm{~Hz}, 3 \mathrm{H}), 4.37(\mathrm{q}, J=7.1 \mathrm{~Hz}, 2 \mathrm{H}), 4.57$ (q, $J=$ $7.1 \mathrm{~Hz}, 2 \mathrm{H}), 7.57(\mathrm{~d}, J=9.6 \mathrm{~Hz}, 1 \mathrm{H}), 7.78(\mathrm{~d}, J=8.5 \mathrm{~Hz}, 2 \mathrm{H}), 8.03(\mathrm{~d}, J=8.5 \mathrm{~Hz}, 2 \mathrm{H}), 8.18\left(\mathrm{dd}, J_{1}=\right.$ $\left.9.6 \mathrm{~Hz}, J_{2}=2.1 \mathrm{~Hz}, 1 \mathrm{H}\right), 9.17\left(\mathrm{bs}\right.$, exchanged by $\mathrm{D}_{2} \mathrm{O}$ addition, $\left.1 \mathrm{H}, \mathrm{NH}\right), 9.84(\mathrm{bs}, 1 \mathrm{H}) .{ }^{13} \mathrm{C}$ NMR $\delta(\mathrm{ppm}) 14.40,14.60,60.75,61.41,99.63,114.55,117.44,122.60,124.18,126.98,131.03$, 137.26, 143.48, 146.59, 166.27. FT-IR $\cup_{\max } 3314,2984,1708,1676,1607,1604,1570,1547$, 1486, 1455, 1434, 1367, 1343, 1313, 1273, 1216, 1177, 1108, 1083, 1019, 857, 762, $750 \mathrm{~cm}^{-1}$ ).

Ethyl 2,9-dioxo-4,9-dihydro-2H-isoxazolo[3,2-b]quinazoline-3-carboxylate (14). To a solution of hydroxylamine hydrochloride $(7.06 \mathrm{~g}, 0.102 \mathrm{~mol})$ in water $(30 \mathrm{~mL})$, sodium bicarbonate $(10.17 \mathrm{~g}, 0.102 \mathrm{~mol})$ was added slowly. Ethanol $(80 \mathrm{~mL})$ was added and the resulting potassium chloride was filtered off. Diethyl (2-ethoxycarbonylphenyl)thiocarbamoylmalonate (12.48g, $34 \mathrm{mmol}$ ) was added to the filtrate and the mixture was refluxed for $24 \mathrm{~h}$. The reaction mixture was cooled to room temperature and acidified with $10 \% \mathrm{HCl}$. The white precipitate was collected and recrystallized from acetone to give the desired product $(7.6 \mathrm{~g}, 81 \%)$ as white needles, m.p. $266-268^{\circ} \mathrm{C}$ (decomposed). Anal. Calc for $\mathrm{C}_{13} \mathrm{H}_{10} \mathrm{~N}_{2} \mathrm{O}_{5}: \mathrm{C}, 56.93 ; \mathrm{H}, 3.64 ; \mathrm{N}$, 10.22\%; found: C, 56.86; H, 3.48; N, 10.34\%. ${ }^{1} \mathrm{H}$ NMR (D 6 -DMSO) $\delta(\mathrm{ppm}) 1.30(\mathrm{t}, J=7.1 \mathrm{~Hz}$, $3 \mathrm{H}), 4.30(\mathrm{q}, J=7.1 \mathrm{~Hz}, 2 \mathrm{H}), 7.45\left(\mathrm{td}, J_{1}=7.6 \mathrm{~Hz}, J_{2}=0.8 \mathrm{~Hz}, 1 \mathrm{H}\right), 7.85\left(\mathrm{td}, J_{1}=7.8 \mathrm{~Hz}, J_{2}=\right.$ $1.5 \mathrm{~Hz}, 1 \mathrm{H}), 8.0(\mathrm{bd}, J=8.2 \mathrm{~Hz}, 1 \mathrm{H}), 8.11\left(\mathrm{dd}, J_{1}=8.0 \mathrm{~Hz}, J_{2}=1.3 \mathrm{~Hz}, 1 \mathrm{H}\right), 12.32(\mathrm{bs}, 1 \mathrm{H}, \mathrm{NH})$. ${ }^{13} \mathrm{C}-\mathrm{NMR}\left(\mathrm{D}_{6}\right.$-DMSO) $\delta$ (ppm) 14.43, 59.82, 74.01, 116.07, 118.39, 125.02, 126.68, 135.24, $138.38,150.27,152.70,161.32,162.21$. FT-IR $v_{\max } 3220,2967,1808,1678,1640,1584,1513$, $1430,1339,1305,1215,1122,1032,929,826,790,756,681 \mathrm{~cm}^{-1}$.

\section{Acknowledgements}

We are grateful to Prof R.H. Prager (Flinders University) for his valuable comments and Prof J. Ipaktschi (Giessen University) for determining the Microanalysis and Mass spectra.

\section{References}

1. Dolesschall, G.A. Tetrahedron Lett. 1988, 29, 6339.

2. Khalafy, J.; Prager, R.H. J.Sci.I.R.Iran. 2000, 11, 32.

3. Khalafy, J.; Prager, R.H, J. Chem. Res(M). 1999, 518. 
4. Khalafy, J.; Molla Ebrahimlo, A.R.; Akbari Dilmaghani, K. J. Chin. Chem. Soc. 2004, $51,1347$.

5. Jeffery, D.; Prager, R.H.; Turner, D.; Dreimanis, M. Tetrahedron 2002, 58, 9965.

6. Worrall, D.E. J.Chem.Soc. 1923, 45, 3092.

7. Worrall,D.E. J.Chem.Soc. 1918, 40, 415.

8. Jeffery, D.W.; Prager, R.H.; Taylor, M.R. Acta Crystallogr. 2001, E57, 0980.

9. Tuteja, N. Pham, T.; Tuteja, R.; Ocham, A.; Falaschi, A. Biochem. Biophys. Res. Commun. 1997, $236,636$.

10. Stiborova, M.; Bicler, C.A.; Wiessler, M.; Frei, E. Biochem. Pharmacol. 2001, 62, 1675.

11. Perrin, D.D.; Armarego,W.L.F. Purification of Laboratory Chemicals; 3rd Edn. Pergamon Press: Oxford, U.K., 1988. 